\title{
Impacto do Programa Academia da Saúde sobre a mortalidade por Hipertensão Arterial Sistêmica no estado de Pernambuco, Brasil
}

\author{
Impact of the Health Gym Program on mortality from Systemic \\ Arterial Hypertension in Pernambuco state, Brazil
}

Bárbara Letícia Silvestre Rodrigues (https://orcid.org/0000-0001-7754-4282) ${ }^{1}$

Rafaela Niels da Silva (https://orcid.org/0000-0001-8065-0780) ${ }^{2}$

Rodrigo Gomes de Arruda (https://orcid.org/0000-0003-3558-6593) ${ }^{3}$

Paloma Beatriz Costa Silva (https://orcid.org/0000-0001-8785-0259) ${ }^{1}$

Daíze Kelly da Silva Feitosa (https://orcid.org/0000-0001-9308-0430) ${ }^{1}$

Flávio Renato Barros da Guarda (https://orcid.org/0000-0002-9214-7784) ${ }^{1}$
${ }^{1}$ Centro Acadêmico de Vitória, Universidade Federal de Pernambuco. R. Alto do Reservatório $\mathrm{s} / \mathrm{n}$, Alto José Leal. 55608-680 Vitória de Santo Antão PE Brasil. barbaralsrodrigues07@ gmail.com

${ }^{2}$ Centro Universitário

Tabosa de Almeida. Caruaru PE Brasil.

${ }^{3}$ Faculdade Nova. Contagem MG Brasil.

\begin{abstract}
The aim of this paper was to analyze the impact of the Health Gym Program (HGP) on the Systemic Arterial Hypertension mortality rate in Pernambuco state, Brazil. This public policy impact analysis used a quasi-experimental approach which consisted of the application of Propensity Score Matching in the years 2010 and 2017. Socioeconomic, demographic, and epidemiological data of 89 municipalities that implemented HGP (treated) and 54 that did not (controls) were collected from the Brazilian Health Data Department, Brazilian Institute of Geography and Statistics, and other databases. The impact of HGP on hypertension mortality rate was estimated through a logit model using the Kernel algorithm. Treated municipalities presented a decrease of $12.8 \%$ in global hypertension mortality rate, $12.5 \%$ in brown-skinned people and $13.1 \%$ in those over 80 years of age. The balancing test attests to the robustness of the estimated model to explain the impact of the program on mortality due to hypertension. The implementation of the program proved to be effective in decreasing the mortality rate in the treated municipalities, indicating that it seems to contribute to controlling the progress of chronic non-communicable diseases. Key words Hypertension, Mortality, Health Policy and Program Evaluation, Health Impact Assessment
\end{abstract}

Resumo O objetivo deste artigo foi avaliar o impacto do Programa Academia da Saúde sobre a mortalidade por Hipertensão Arterial Sistêmica no estado de Pernambuco, Brasil. Este estudo quase-experimental configura-se como uma avaliação de impacto de políticas públicas, desenvolvida através da aplicação do método do Pareamento por Escore de Propensão, nos anos de 2010 e 2017. Utilizou-se dados socioeconômicos, demográficos e epidemiológicos dos 89 municípios que implantaram o programa (tratados) e de outros 52 que não implantaram (controles). Os dados são oriundos do Departamento de Informática do SUS, Instituto Brasileiro de Geografia e Estatística e outras bases. O impacto do programa foi estimado através de um modelo logit, com o uso do algoritmo de pareamento Kernel. Os municípios tratados tiveram uma diminuição global de 12,8\% na taxa de mortalidade por hipertensão, de 12,5\% entre as pessoas de cor parda e de 13,1\% em maiores de 80 anos. O teste de balanceamento atesta a robustez do modelo para explicar o impacto do programa sobre a mortalidade. A implementação do programa se mostrou efetiva para diminuir a taxa de mortalidade nos municípios tratados, indicando que o mesmo parece estar contribuindo para controlar o avanço das doenças crônicas não transmissiveis.

Palavras-chave Hipertensão, Mortalidade, Avaliação de Programas e Projetos de Saúde, Avaliação do Impacto na Saúde 


\section{Introdução}

Evidências a respeito da carga global de Hipertensão Arterial apontam que $26,4 \%$ da população adulta em todo o mundo era hipertensa no ano 2000 e que até 2025 essa prevalência deve aumentar em $60 \%$, sendo esse aumento mais expressivo nos países em desenvolvimento ${ }^{1}$.

A Hipertensão Arterial Sistêmica (HAS) tem origem multifatorial e é caracterizada pelo aumento dos níveis pressóricos sanguíneos para valores iguais ou superiores a $140 \mathrm{mmHg}$ para a pressão sistólica e $90 \mathrm{mmHg}$ para a diastólica ${ }^{2}$, configura-se como um fator de risco para doenças cerebrovasculares e cardiovasculares ${ }^{3}$ e que pode ser agravada por fatores biológicos (dislipidemia, obesidade abdominal, intolerância à glicose $\mathrm{e}$ diabetes) ${ }^{2}$, comportamentais (tabagismo, alimentação e inatividade física $)^{3}$ e socioeconômicos (renda, escolaridade, Índice de Desenvolvimento Humano, Produto Interno Bruto - PIB per capita - dos Municípios e acesso a serviços de saúde $)^{4-6}$.

A HAS é a doença com maior impacto sobre a morbimortalidade no Brasil ${ }^{7}$, com prevalência de $32,6 \%$ entre os adultos, acima de $60 \%$ nos idosos ${ }^{8}$ e taxa de mortalidade de 0,87 óbitos para cada 10 mil adultos no Brasil ${ }^{8} ; 2,56$ para a região Nordeste (maior entres as regiões) e 2,13 no estado de Pernambuco (13a no país). Entre os principais preditores da mortalidade por HAS no Brasil estão o aumento da idade, a cor parda ${ }^{9} \mathrm{e}$ o nível de escolaridade da população ${ }^{10}$. Além disso, os óbitos por HAS têm associação inversa com variáveis socioeconômicas dos municípios ${ }^{6}$ e com o nível de atividade física dos indivíduos ${ }^{11-13}$.

Evidências de estudos com diferentes populações e com a população brasileira apontam que a prática regular de atividade física diminui o risco de morte por HAS e por outras doenças crônicas não transmissiveis $(\mathrm{DCNT})^{11-13}$, o que tem levado as autoridades sanitárias a investir na implantação de políticas e programas de incentivo à adoção de estilos de vida mais ativos e saudáveis na população ${ }^{14,15}$.

Com o intuito de integrar a prática de atividade física à agenda das políticas de saúde do país, o Ministério da Saúde instituiu em 2011 o Programa Academia da Saúde (PAS), com o objetivo de contribuir para a promoção da saúde através do financiamento federal e cofinanciamento municipal para a construção e/ou reforma de espaços públicos com infraestrutura (chamados de polos) e profissionais qualificados para a realização de atividades de promoção da saúde e produção do cuidado no campo da Atenção Primária à Saúde (APS) ${ }^{16,17}$.
O PAS é considerado como um programa estratégico para a execução das políticas nacionais de promoção da saúde e tem entre os seus objetivos específicos a proposição de aumentar o nível de atividade física da população ${ }^{17}$. Para mais, além da sua proposta de promover saúde, agindo na prevenção e controle das doenças crônicas, influenciando sobre os condicionantes e determinantes da saúde, o PAS tem papel importante na melhoria da qualidade de vida da população, pois lida tanto com aspectos fisiológicos quanto sociais do processo saúde-doença ${ }^{18}$.

As atividades do PAS compõem o escopo da Política Nacional de Promoção da Saúde (PNPS) e da Política Nacional de Atenção Básica (PNAB), e o programa é apontado como uma das ações de prevenção e controle que integram o Plano de Ações Estratégicas para o Enfrentamento das Doenças Crônicas Não Transmissíveis (DCNT) $)^{16-19}$.

Registros presentes no Cadastro Nacional de Estabelecimentos de Saúde (CNES) apontam que a região Nordeste do país concentrou no ano de 2017 um total de 917 polos do programa, seguida da região Sudeste (527), Sul (390), Centro-Oeste (193) e da região Norte com 189 polos do $\mathrm{PAS}^{20}$.

O estado de Pernambuco foi um dos pioneiros na implantação do PAS, tendo iniciado suas atividades desde $2011^{21}$. Até 2017 o estado havia implantado 246 polos do em seu território, o que corresponde a $11,10 \%$ do total de unidades implantadas no país ${ }^{20}$.

Nesse cenário, avaliação de impacto do PAS em Pernambuco pode ser considerada uma ferramenta de gestão que permite identificar potencialidades e fragilidades do programa, além de contribuir para o processo de tomada de decisão de trabalhadores, gestores e financiadores dessa intervenção ${ }^{22}$. Dessa forma, o objetivo deste estudo é analisar o impacto do programa Academia da Saúde sobre a mortalidade por Hipertensão Arterial Sistêmica no estado de Pernambuco.

A escolha de avaliar a mortalidade sobre Hipertensão Arterial se deve ao fato de que um conjunto robusto de evidências aponta a associação entre a inatividade física e o risco de adoecimento e morte por essa doença $a^{11-13}$, e pelo fato de que o PAS tem como um dos seus objetivos específicos o aumento do nível de atividade física da população ${ }^{16}$, configurando-se como uma das estratégias de prevenção e controle de doenças crônicas previstas nas políticas nacionais de Atenção Básica, de Promoção da Saúde e no Plano de Ações Estratégicas para o Enfrentamento das Doenças Crônicas Não Transmissíveis no Brasil ${ }^{16,23,24}$. 


\section{Métodos}

\section{Caracterização do estudo}

Este estudo caracteriza-se como uma avaliação de impacto de políticas públicas, desenvolvido através de uma abordagem quase-experimental que consiste na aplicação do método do pareamento por escore de propensão (aqui designado como Propensity Score Matching - PSM) para estimar o Efeito Médio do Tratamento sobre os Tratados (Average Treatment Effect for the Treated - ATT). O ATT é caracterizado neste estudo pelo efeito do Programa Academia da Saúde sobre a taxa de mortalidade (por 10 mil habitantes) por Hipertensão Arterial Sistêmica no estado de Pernambuco.

\section{Amostragem e base de dados}

Os dados sobre a presença dos polos do PAS nos municípios pernambucanos foram coletados no site do Cadastro Nacional de Estabelecimentos de Saúde (CNES), do Departamento de Informática do SUS (DATASUS).

Os dados epidemiológicos referem-se aos óbitos e seus respectivos extratos por sexo, faixa etária e raça/cor (oriundos do Sistema de Informação sobre Mortalidade - SIM), à taxa de leitos hospitalares para cada 1.000 habitantes (extraídas do CNES), e à Cobertura da Atenção básica (sítio do Sistema de Informação e Gestão da Atenção Básica - e-Gestor). Como variáveis demográficas o estudo tomou como referência a população geral por município, proporção da população feminina e masculina, proporção de residentes por faixas etárias de 40 a 49 anos, 50 a 59 anos, 60 a 69 anos, 70 a 79 anos e de 80 anos e mais (coletadas no site do Instituto Brasileiro de Geografia e Estatística - IBGE), o Índice de Desenvolvimento Humano (IDH) (na Base de Dados do Estado BDE), e os Índice FIRJAN de desenvolvimento Municipal (IFDM), Índice FIRJAN de desenvolvimento relacionado à saúde e relacionado à educação. As variáveis socioeconômicas utilizadas para este estudo foram PIB per capita e o Índice de FIRJAN para emprego e renda. Os dados sobre o índice de FIRJAN e PIB per capita foram coletados a partir dos sites da Federação das Indústrias do Estado do Rio de Janeiro (FIRJAN).

Dos 185 municípios em todo o estado, este estudo considerou como tratados os 89 que implantaram o Programa Academia da Saúde no ano de 2011 e como controles os 52 municípios que não implantaram. Foram excluídos da amos- tra os municípios que implantaram o programa após o ano de 2011 e os que o implantaram nesse ano e cancelaram as suas atividades nos anos subsequentes. Os dados foram coletados tomando como referência os anos de 2010 (ano anterior à implantação) e 2017 (seis anos após o início da implantação do PAS no estado de Pernambuco).

A variável resultado para este estudo é o logaritmo natural da taxa de mortalidade por Hipertensão Arterial por 10 mil habitantes, por local de residência do indivíduo. Esses logaritmos são utilizados no campo da econometria, sobretudo quando as relações entre as variáveis dependentes e independentes não se dão de forma linear ${ }^{25}$.

Considerando que alguns valores das taxas de mortalidade por HAS eram iguais a zero, utilizou-se o artifício de somar uma unidade ao valor original da taxa antes de realizar a conversão para o logaritmo natural, conforme recomendado por Wooldridge ${ }^{25}$.

\section{Covariáveis de controle}

As covariáveis deste estudo foram selecionadas a partir de evidências científicas acerca da relação de confundimento que elas podem exercer sobre a relação entre exposição e o desfecho de interesse. Neste caso, o modelo epidemiológico que orientou a seleção das variáveis explicativas tomou como referência os estudos de Guimarães et al. ${ }^{7}$ e Santos et al. ${ }^{9}$, os quais apontam fatores associados à mortalidade por Hipertensão Arterial na população brasileira ${ }^{7,9}$.

\section{Propensity Score Matching e impacto do Programa Academia da Saúde sobre a mortalidade por HAS}

Considerando que a implementação do PAS nos municípios se deu por adesão e que não houve aleatoriedade na composição dos grupos expostos e não expostos a essa intervenção, a amostra que compõe este estudo poderia estar sujeita à problemas como o viés de seleção, e sensível à multidimensionalidade dos fatores que determinam a probabilidade de implantação dessa política. Para minimizar esses problemas, este estudo utilizou o método do pareamento por escore de propensão (PSM), o qual assemelha os dois grupos em relação algumas características socioeconômicas, demográficas e epidemiológicas e calcula a probabilidade de os municípios aderirem ao programa com base nesses perfis, criando um cenário contrafactual que permite comparar os municípios tratados e controles ${ }^{26}$. 
O PSM caracteriza-se como um método quase-experimental que permite a formação de grupos (de indivíduos ou outras unidades agregadas) com características semelhantes, mas que diferem entre si em relação à exposição (ou não) a uma determinada intervenção ${ }^{27,28}$.

$\mathrm{O}$ método foi desenvolvido para resolver o problema da multidimensionalidade do pareamento e consiste em identificar unidades nãotratadas que sejam similares às unidades tratadas e comparar as médias no resultado, procurando identificar, por meio da seleção de características observáveis entre estes dois grupos, o impacto do tratamento (PAS). O propensity score matching configura-se como um importante recurso de pareamento utilizado para avaliação de políticas públicas $^{26,27}$.

Os procedimentos de pareamento utilizam um escore balanceado, computado a partir de um modelo de regressão (logit ou probit) que utiliza uma variável dependente tipo dummy que assume o valor um se o indivíduo (ou unidade) foi exposto à política em análise, ou zero, caso contrário.

A escolha entre os modelos logit e probit foi realizada por meio do Critério da Informação de Akaike (AIC) e do Critério de Informação Bayesiano (BIC), considerando os menores valores encontrados em ambos os critérios como referência de melhor ajustamento do modelo ${ }^{29}$.

O PSM é definido como a probabilidade do indivíduo (município) ser beneficiário do programa, dadas suas características (socioeconômicas, demográficas e epidemiológicas).

Através da estimativa do PSM são apontados subgrupos dentro do grupo de controle, com probabilidades semelhantes às dos municípios do grupo de intervenção. Em seguida, realiza-se o balanceamento das variáveis, o qual permite testar para cada bloco do propensity score, se a média de cada variável utilizada no modelo não diferia entre municípios beneficiários e não beneficiários do PAS.

Após essa etapa, um número final de blocos foi definido e prosseguiu-se com o cálculo do Efeito Médio do Tratamento sobre os Tratados (ATT) por meio da testagem dos algoritmos de pareamento. Esses testes visam construir um cenário contrafactual a partir da média ponderada do número de unidades de controle com cada unidade de tratamento ${ }^{28}$. Por meio desse método, cada unidade do grupo tratado foi pareada com a unidade do grupo controle com valor do propensity score mais próximo. O ATT foi determinado para avaliar o impacto do Programa Academia da Saúde sobre a mortalidade por Hipertensão Arterial para cada um dos grupos de municípios (tratados e controles).

\section{Análise dos dados}

Foram adotados procedimentos de estatística descritiva (frequências, médias e desvios-padrão) para caracterizar o perfil socioeconômico, demográfico e epidemiológico dos municípios tratados e controles antes do pareamento. Para a comparação das médias e desvios-padrão das variáveis relativas aos expostos e não expostos à política em análise (Programa Academia da Saúde) e o respectivo cálculo do tamanho de efeito, foi utilizada a medida $D$ de Cohen.

Para a estimativa do PSM foram testados modelos de regressão para dados binários, usando as funções de ligação logit e probit ${ }^{30}$, para determinar a probabilidade de participação dos municípios no PAS, dadas as características socioeconômicas, demográficas e epidemiológicas dos municípios que compuseram a amostra, através de um vetor de características do período anterior à exposição ao programa $\left(\mathrm{X}_{\mathrm{i}, 1}\right)^{31}$, o qual é dado por:

$$
\mathrm{P}\left(P \mathrm{AS}_{i}^{0}=1\right)=\emptyset\left(\beta X_{i^{\prime}-1}\right)
$$

Onde PAS $_{i}$ é uma variável dummy que assume o valor 1 (tratado) se o i-ésimo município foi exposto ao PAS e o valor 0 (controle) se não foi exposto. $\emptyset$ é uma função de distribuição acumulada do tipo logit ou probit, $\mathrm{X}_{\mathrm{i}, 1}$ é um vetor de $\mathrm{k}$ variáveis explanatórias ponderadas pelo inverso da probabilidade de tratamento e $\beta$ é um vetor de parâmetros associados a essas variáveis.

A probabilidade de o município ser tratado, dado o conjunto de características $X$, é denominada de escore de propensão, o qual é definido por:

$\hat{\mathrm{P}}(\mathrm{X})=\mathrm{P}\left(\mathrm{PAS}_{i}^{0}=1 \mid X_{i,-1}\right)$

Para calcular esse escore, utilizou-se um conjunto de variáveis socioeconômicas, demográficas e epidemiológicas que potencialmente poderiam interferir na disposição de um município aderir ao PAS.

$\mathrm{Na}$ etapa seguinte, os escores de propensão estimados foram utilizados para computar os pesos necessários para balancear os municípios no grupo de controle, de modo que, em sua média, esses se tornem semelhantes aos tratados. Para tanto, foram testados os métodos do vizinho mais próximo (1:5), com e sem reposição, pareamento de Kernel e pareamento radial, de modo a identificar a melhor forma de realizar o pareamento $^{28}$. 
Para testar a robustez do modelo foi realizado o teste de balanceamento (pstest), o qual buscou verificar similaridades estatísticas entre as variáveis do pareamento e a redução do viés padronizado antes e depois, ambas ao nível de $5 \% \%^{26,27}$.

Todas as análises foram realizadas no software Stata versão 15.0, considerando o delineamento complexo da amostra. Utilizou-se o aplicativo do Stata "psmatch2" para o cálculo do propensity score e do ATT, adotando um nível de significância de $5 \%$ que foi para todos os testes estatísticos.

Visando evitar comparações entre municípios que iniciaram as atividades do PAS em períodos distintos, e que nesses casos estariam sujeitas aos potenciais efeitos do programa em diferentes momentos no tempo, foram excluídas da análise as cidades que iniciaram as atividades do PAS há menos de seis anos.

\section{Resultados}

Os resultados são apresentados em três seções. $\mathrm{Na}$ primeira, apresenta-se a análise descritiva das características socioeconômicas, demográficas e epidemiológicas dos municípios que compõem os grupos de tratados e controles, antes da realização do pareamento. Na segunda parte, descreve-se o perfil da mortalidade da amostra, estratificado entre tratados e controles antes da implementação do PAS. A terceira seção apresenta os resultados da estimação do modelo de avaliação do impacto do PAS sobre a mortalidade por HAS e na quarta parte apresentam-se os resultados referentes ao impacto do PAS sobre a taxa de mortalidade por Hipertensão Arterial no estado de Pernambuco.

\section{Características socioeconômicas, demográficas e epidemiológicas dos municípios}

No estado de Pernambuco foram registrados um total de 35.493 óbitos por todas as causas, sendo 783 (2,20\%) por HAS no ano de 2010, enquanto em 2017 foram registrados 42.134 óbitos, sendo $728(1,72 \%)$ por HAS. A taxa de mortalidade por HAS foi de 1,59 e 1,60 óbitos para cada grupo de 10 mil habitantes para os anos de 2010 e 2017, respectivamente.

A análise descritiva do perfil epidemiológico e assistencial dos municípios tratados e controles antes da implantação do PAS (2010) demonstrou que os municípios que viriam a implantar o programa em 2011, já tinham maiores taxas de mortalidade geral e de óbitos em pessoas maiores de 50 anos e maior taxa de leitos, além de menor taxa de mortalidade por HAS e menor cobertura da Atenção Básica que os municípios considerados controles (Tabela 1).

No que cabe às características socioeconômicas, conforme descrito na Tabela 1, os municípios que vieram a implantar o PAS em 2011 já apresentavam em 2010 melhores indicadores de PIB, Índice de Gini, IFDM Geral, IFDM para a educação, taxa de desemprego e IDH.

\section{Perfil da mortalidade por Hipertensão Arterial nos municípios}

Em relação à taxa de mortalidade por HAS no ano de 2010, observou-se que os municípios avaliados apresentavam maior taxa de mortalidade por HAS na população feminina em relação à população masculina. No que se refere à variável raça/cor, as maiores taxas estavam entre os pardos, e com relação à faixa etária observou-se que a taxa de mortalidade por HAS, tanto nos municípios tratados, quanto nos controles, foi maior nos indivíduos mais velhos, com valores mais expressivos na população acima dos 60 anos (Tabela 2).

\section{Estimação do modelo de avaliação do impacto do PAS sobre a mortalidade por HAS}

Para realizar o Pareamento por Escore de Propensão foram estimados modelos logit e probit, considerando as variáveis socioeconômicas, demográficas e da rede de atenção à saúde dos municípios que compõe a amostra. O modelo logit foi selecionado por apresentar menores valores do AIC e BIC que o modelo probit e o algoritmo de Kernel mostrou a melhor significância estatística e foi escolhido para avaliar o impacto do PAS sobre a mortalidade por HAS. Os resultados do modelo logit são apresentados na Tabela 3 , juntamente com os algoritmos de pareamento.

O Pareamento por Escore de Propensão foi estimado através do modelo logit, considerando variáveis socioeconômicas e de saúde dos municípios que constituem a amostra. A Tabela 4 descreve o modelo usado para realizar o pareamento por escore de propensão.

A análise demonstra que as variáveis que melhor explicam a probabilidade de um município aderir ao PAS foram o Produto Interno Bruto, a cobertura da atenção básica do município, a taxa de leitos hospitalares por 1.000 habitantes, 
Tabela 1. Características socioeconômicas, demográficas e epidemiológicas dos municípios tratados e controles. Pernambuco, 2010.

\begin{tabular}{|c|c|c|c|c|c|c|c|}
\hline \multirow{2}{*}{ Variáveis } & \multicolumn{2}{|c|}{ Controle } & \multicolumn{2}{|c|}{ Tratado } & \multirow{2}{*}{$\begin{array}{l}\text { Diferença } \\
\text { de médias }\end{array}$} & \multirow{2}{*}{$\begin{array}{c}\text { d- } \\
\text { Cohen }\end{array}$} & \multirow{2}{*}{ IC95\%* } \\
\hline & Média & DP & Média & DP & & & \\
\hline $\begin{array}{l}\text { Proporção de homens na } \\
\text { população }\end{array}$ & 0,508 & 0,008 & 0,505 & 0,008 & 0,003 & 0,408 & $0,062-0,753$ \\
\hline $\begin{array}{l}\text { Proporção de mulheres na } \\
\text { população }\end{array}$ & 0,512 & 0,007 & 0,515 & 0,008 & $-0,003$ & $-0,371$ & $-0,715--0,026$ \\
\hline $\begin{array}{l}\text { Proporção de pessoas } 40 \text { a } 49 \\
\text { anos }\end{array}$ & 0,118 & 0,071 & 0,111 & 0,013 & 0,007 & 0,159 & $-0,182-0,502$ \\
\hline $\begin{array}{l}\text { Proporção de pessoas } 50 \text { a } 59 \\
\text { anos }\end{array}$ & 0,083 & 0,055 & 0,078 & 0,010 & 0,005 & 0,142 & $-0,200-0,484$ \\
\hline $\begin{array}{l}\text { Proporção de pessoas } 60 \text { a } 69 \\
\text { anos }\end{array}$ & 0,061 & 0,044 & 0,059 & 0,010 & 0,002 & 0,061 & $-0,281-0,403$ \\
\hline $\begin{array}{l}\text { Proporção de pessoas } 70 \text { a } 79 \\
\text { anos }\end{array}$ & 0,036 & 0,025 & 0,035 & 0,008 & 0,001 & 0,047 & $-0,294-0,390$ \\
\hline Proporção de pessoas $>80$ anos & 0,018 & 0,014 & 0,018 & 0,005 & 0 & $-0,040$ & $-0,382-0,302$ \\
\hline Taxa óbitos $>50$ anos /1.000 hab & 4,28 & 1,23 & 4,54 & 1,11 & $-0,26$ & $-0,222$ & $-0,565-0,120$ \\
\hline $\begin{array}{l}\text { Taxa de mort Geral (por } 1.000 \\
\text { hab) }\end{array}$ & 5,98 & 1,312 & 6,22 & 1,24 & $-0,24$ & $-0,190$ & $-0,532-0,152$ \\
\hline $\begin{array}{l}\text { Taxa de mort por HAS/ } 10.000 \\
\text { hab }\end{array}$ & 1,85 & 1,68 & 1,44 & 1,34 & 0,41 & 0,274 & $-0,069-0,617$ \\
\hline Taxa de Leitos/1.000 habitantes & 1,407 & 0,954 & 1,600 & 1,130 & $-0,193$ & $-0,180$ & $-0,522-0,162$ \\
\hline $\begin{array}{l}\text { Cobertura da Atenção Básica } \\
(\%)\end{array}$ & 91,4 & 15,0 & 85,8 & 19,4 & 5,6 & 0,311 & $-0,032-0,655$ \\
\hline Índice de Gini & 0,514 & 0,043 & 0,521 & 0,045 & $-0,007$ & $-0,159$ & $-0,501-0,183$ \\
\hline IFDM Geral & 0,576 & 0,066 & 0,580 & 0,078 & $-0,004$ & $-0,052$ & $-0,394-0,289$ \\
\hline $\begin{array}{l}\text { Produto interno bruto (per } \\
\text { capita) }\end{array}$ & $5.872,50$ & $2.796,10$ & $6.463,50$ & $4.156,10$ & $-591,00$ & $-0,159$ & $-0,501-0,183$ \\
\hline $\begin{array}{l}\text { Índice de Desenvolvimento } \\
\text { Humano }\end{array}$ & 0,586 & 0,044 & 0,602 & 0,048 & $-0,016$ & $-0,344$ & $-0,688-0,000$ \\
\hline Taxa de desemprego & 10,69 & 5,43 & 8,24 & 3,71 & 2,45 & 0,554 & $0,204-0,901$ \\
\hline IFDM Educação & 0,569 & 0,074 & 0,609 & 0,633 & $-0,04$ & $-0,593$ & $-0,941--0,242$ \\
\hline
\end{tabular}

Fonte: Elaborado pelos autores a partir dos dados do SIM, DATASUS, BDE e IBGE, utilizando STATA.

a taxa de desemprego e o índice de FIRJAN para a educação, sendo que as duas últimas se mostraram estatisticamente significantes ao nível de $5 \%$. Neste sentido, observou-se que a chance de um município aderir/implantar o PAS é diretamente proporcional ao aumento no indicador de educação e inversamente proporcional à taxa de desemprego.

O teste de robustez do modelo mostrou que o pareamento atendeu à propriedade de balanceamento, pois não foram observadas similaridades estatísticas entre as variáveis do pareamento (todas com p>0,05). Além disso, no que cabe à redução do viés padronizado antes/depois, verificouse que todas as variáveis apresentaram redução do percentual de bias de pelo menos $5 \%$.

\section{Impacto do PAS sobre a taxa de mortalidade por Hipertensão Arterial}

O pareamento por escore de propensão foi usado neste estudo com o objetivo de tornar os grupos tratados e de controle semelhantes, considerando suas características observáveis descritas na Tabela 5 .

$\mathrm{Na}$ análise do PSM apresentada na Tabela 5 , observa-se que a presença do PAS ocasionou uma redução de $12,8 \%$ na taxa de mortalidade por hipertensão arterial e que essa redução foi maior e estatisticamente significante nas pessoas de cor parda e em maiores de 80 anos. 
Tabela 2. Taxa de mortalidade por HAS para cada 10 mil habitantes nos municípios tratados e controles. Pernambuco, 2010.

\begin{tabular}{lcccccc}
\hline \multirow{2}{*}{ Extratos } & \multicolumn{2}{c}{ Tratados } & \multicolumn{2}{c}{ Controles } & \multirow{2}{*}{ d-Cohen } & \multirow{2}{*}{ IC95\% $^{*}$} \\
\cline { 2 - 5 } & Total & DP & Total & DP & & \\
\hline Feminino & 0,72 & 0,74 & 0,84 & 0,81 & 0,156 & $-0,184-0,495$ \\
Masculino & 0,66 & 0,79 & 0,99 & 1,14 & 0,342 & $-0,000-0,683$ \\
Brancos & 0,48 & 0,68 & 0,54 & 0,77 & 0,086 & $-0,256-0,428$ \\
Pretos & 0,09 & 0,21 & 0,16 & 0,32 & 0,272 & $-0,071-0,615$ \\
Pardos & 0,75 & 0,84 & 1,06 & 1,24 & 0,314 & $-0,029-0,658$ \\
Outra raça/cor & 0,03 & 0,13 & 0,02 & 0,12 & $-0,134$ & $-0,476-0,208$ \\
40 a 49 anos & 0,06 & 0,19 & 0,06 & 0,18 & $-0,044$ & $-0,386-0,297$ \\
50 a 59 anos & 0,09 & 0,22 & 0,15 & 0,33 & 0,211 & $-0,132-0,553$ \\
60 a 69 anos & 0,18 & 0,33 & 0,25 & 0,43 & 0,181 & $-0,161-0,523$ \\
70 a 79 anos & 0,36 & 0,52 & 0,45 & 0,53 & 0,172 & $-0,170-0,515$ \\
80 anos e mais & 0,67 & 0,77 & 0,91 & 1,03 & 0,272 & $-0,071-0,615$ \\
\hline
\end{tabular}

*IC95\%: Intervalo de Confiança 95\% da medida $D$ de Cohen.

Fonte: Elaborado pelos autores a partir dos dados do SIM, DATASUS, BDE e IBGE, utilizando STATA.

Tabela 3. Teste dos algoritmos de pareamento do PAS sobre a mortalidade por Hipertensão Arterial, através do método Pareamento por escore de propensão.

\begin{tabular}{lcccc}
\hline \multicolumn{5}{c}{ Diferença das médias } \\
\hline \multicolumn{1}{c}{ Mortalidade por $\mathbf{H A S}^{\mathbf{a}}$} & Tratado & Controle & Diferença & Valor-t \\
\hline Sem algoritmo & 0,820 & 0,948 & $-0,127$ & $-1,43^{* *}$ \\
Radial & 0,820 & 0,862 & $-0,041$ & $-1,03$ \\
Vizinho mais próximo & 0,820 & 0,956 & $-0,136$ & $-1,79^{\star}$ \\
Kernel & 0,820 & 0,948 & $-0,128$ & $-1,73^{\star}$ \\
\hline
\end{tabular}

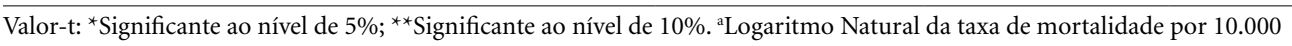

habitantes.

Fonte: Elaborado pelos autores a partir dos dados do SIM, DATASUS, BDE e IBGE, utilizando STATA.

Tabela 4. Modelo logit de participação no Programa Academia da Saúde e teste de balanceamento do modelo. Pernambuco, 2010 e 2017.

\begin{tabular}{|c|c|c|c|c|}
\hline \multicolumn{5}{|c|}{ Modelo Logit kernel } \\
\hline Variáveis & Coef & Erro-padrão & Valor-p & IC95\% \\
\hline PIB & 0,000 & 0,000 & 0,504 & $-0,000-0,000$ \\
\hline Cobertura $\mathrm{AB}$ & $-1,653$ & 0,893 & 0,064 & $-3,403-0,096$ \\
\hline Taxa leitos & 0,080 & 0,137 & 0,556 & $-0,188-0,349$ \\
\hline Taxa desemprego & $-0,122$ & 0,030 & $<0,001$ & $-0,181--0,062$ \\
\hline IFDM Educação & 1,788 & 1,816 & 0,001 & $2,420-9,542$ \\
\hline Constante & $-0,872$ & 1,264 & 0,490 & $-3,351-1,606$ \\
\hline \multicolumn{5}{|c|}{ Balanceamento do modelo } \\
\hline \multirow{2}{*}{ Variáveis } & \multicolumn{2}{|c|}{ Média } & \multirow{2}{*}{$\%$ Bias } & \multirow{2}{*}{ Valor-p } \\
\hline & Tratados & Controles & & \\
\hline PIB & 8534,1 & 8253,2 & 5,2 & 0,610 \\
\hline Cobertura $\mathrm{AB}$ & 0,899 & 0,910 & $-7,2$ & 0,517 \\
\hline Taxa leitos & 1,527 & 1,436 & 9,1 & 0,374 \\
\hline Taxa desemprego & 8,246 & 7,659 & 12,7 & 0,164 \\
\hline Educação & 0,655 & 0,646 & 11,3 & 0,284 \\
\hline
\end{tabular}

IC95\%: Intervalo de Confiança 95\%. 


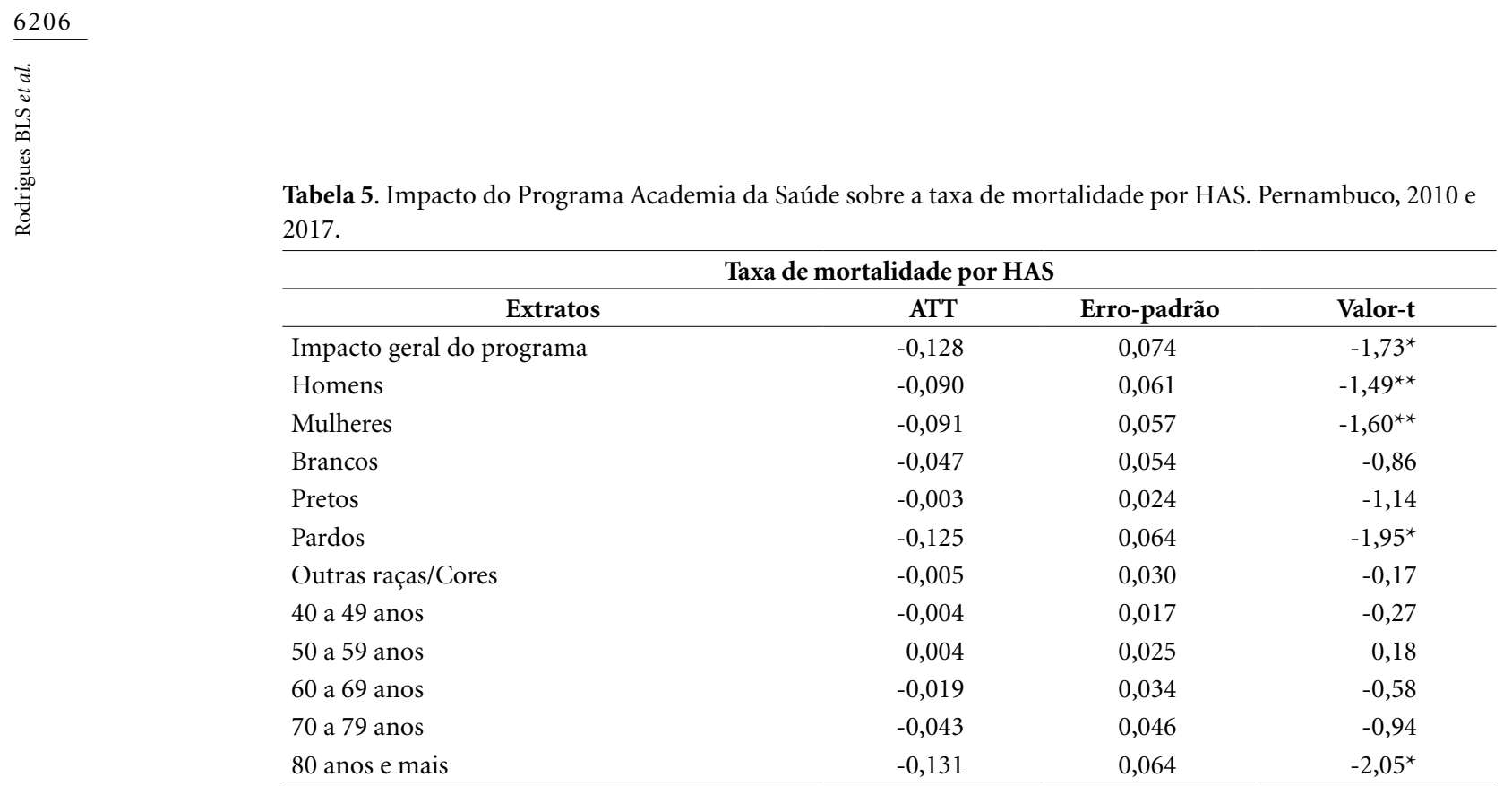

ATT=Efeito Médio do Tratamento sobre os Tratados. Valor-t: *Significante ao nível de 5\%; ${ }^{*}$ Significante ao nível de $10 \%$.

Fonte: Elaborado pelos autores a partir dos dados do SIM, DATASUS, BDE e IBGE, utilizando STATA.

\section{Discussão}

Neste estudo observou-se que a HAS foi responsável por cerca de dois por cento das mortes por todas as causas no estado em 2010 e em 2017, com taxa de mortalidade por mil habitantes praticamente igual para os dois anos avaliados, corroborando os resultados de estudos que apontam estabilidade na mortalidade por essa causa ao longo da última década9 .

A análise descritiva do perfil socioeconômico, epidemiológico e assistencial dos municípios tratados e controles antes da implementação do PAS, reiteram resultados dos sistemas de informações em saúde e de estudos epidemiológicos, os quais apontam que a taxa de leitos por mil habitantes em Pernambuco é ligeiramente menor que a média nacional $(1,67)^{32}$ e que a cobertura da atenção básica encontrada neste estudo foi maior que a média do Brasil $(51,8 \%)^{33}$. Ressalta-se, entretanto, que as diferenças observadas no perfil dos municípios antes do PSM foram eliminadas através da seleção de pares de tratados e controles que apresentassem características e probabilidades de implementação do PAS semelhantes ${ }^{26}$.

Os achados relacionados ao perfil da mortalidade por sexo neste estudo confrontam os resultados de pesquisas que apontam que a mortalidade por HAS é mais prevalente em homens. Em estudo que avaliou as variações e diferenciais da mortalidade por doença cardiovascular no Brasil,
Brant et al. ${ }^{34}$ apontam que a mortalidade por doenças hipertensivas foi maior em indivíduos do sexo masculino entre 1990 e 2015.

Com relação à variável raça-cor, nosso estudo demonstrou maior taxa de mortalidade na população preta e parda, corroborando resultados de outros estudos sobre mortalidade por doenças hipertensivas, que apontam maiores riscos em indivíduos não brancos, do que em brancos ${ }^{35}$. Nessa perspectiva, as altas taxas de mortalidade por hipertensão em indivíduos de cor preta (tanto em Pernambuco, quanto no Brasil), podem ser justificadas pelas condições socioeconômicas, que estão intrinsicamente ligadas às desigualdades de oportunidades sociais ao longo da vida nesses subgrupos ${ }^{36}$.

Neste estudo, observamos que a taxa mortalidade por HAS é diretamente proporcional ao aumento da idade, corroborando com estudo que aponta que o incremento progressivo da idade aumenta as chances da ocorrência de óbitos por $\mathrm{HAS}^{9}$.

No que se refere à estimação do modelo de avaliação do impacto do PAS sobre a taxa de mortalidade por hipertensão, foram testados modelos do tipo probit e logit, sendo este último o escolhido para mensurar a probabilidade de um município implantar o PAS. O modelo logit é o modelo de regressão mais amplamente utilizado em pesquisas no campo da avaliação de impacto de políticas públicas ${ }^{37}$. Além disso, se mostrou mais adequado aos dados e apresentou menores 
valores para os Critérios da Informação de Akaike (AIC) e de Informação Bayesiano (BIC) ${ }^{29}$.

Os resultados dos testes dos algoritmos de pareamento mostraram que o método do vizinho mais próximo apresentou resultados ligeiramente melhores que os do pareamento de Kernel, tanto para o impacto do PAS sobre a mortalidade, quanto na significância estatística do efeito do tratamento sobre os tratados (ATT). Entretanto, como os valores foram próximos, optou-se pelo uso do algoritmo de Kernel por ser mais eficiente e produzir variâncias mais baixas, quando comparado com outras estratégias de pareamento ${ }^{38}$.

O pareamento através do algoritmo de Kernel tornou a probabilidade condicional de implantar polos do PAS mais semelhantes entre os grupos tratado e controle, minimizando o viés de seleção e indicando que o PSM foi bem sucedido ${ }^{39,40}$.

As variáveis que compuseram o modelo de avaliação do impacto do PAS sobre a mortalidade por HAS (PIB, cobertura da atenção básica, a taxa de leitos hospitalares, taxa de desemprego e o índice de FIRJAN para a educação) corroboram com achados da literatura que relatam a influência da renda, estrutura da rede de atenção à saúde e escolaridade sobre a taxa de óbito por HAS ${ }^{41,42}$. Além disso, o modelo proposto atendeu às propriedades de balanceamento, as quais indicam que o PSM consegue equilibrar as características observáveis dos grupos tratado e controle ${ }^{39,40}$.

O teste de balanceamento demonstrou ainda que a média de cada variável utilizada no modelo não diferia entre os tratados e controles, indicando que a participação dos municípios no PAS não depende dos resultados encontrados, ou seja, a mortalidade não é determinante da participação (ou não) no programa, mas apenas determinada por ele $e^{26,43}$.

Antes da implementação do PAS em 2010 já existia uma discrepância no perfil da mortalidade por HAS entre os municípios avaliados, entretanto, após a implantação do programa verificou-se que municípios beneficiários (tratados) apresentaram uma taxa de óbitos por HAS 12,8\% menor, em comparação aos municípios não beneficiários do programa (controles).

O impacto do programa sobre pessoas de cor preta e parda, bem como as de maior idade, reitera resultados de estudos que apontam os efeitos de programa similar ao PAS no aumento dos níveis de atividade física em pessoas não brancas e indivíduos mais velhos ${ }^{44,45}$.

A relação entre a presença do PAS e a mortalidade por hipertensão pode estar relacionada à adoção de estilos de vida mais ativos e saudáveis por parte da população dos municípios beneficiários, o que nos permite inferir que o objetivo específico do PAS de aumentar a qualidade do cuidado e o nível de atividade física da população ${ }^{17,45}$ e a sua diretriz de configurar-se como estratégia de promoção da saúde, produção do cuidado e prevenção de doenças crônicas ${ }^{18}$ está sendo alcançado nos municípios tratados. Por outro lado, evidências apontam a falta de metas claramente definidas ${ }^{16}$ e de protocolos de ação ${ }^{24}$, os quais podem ampliar excessivamente as possibilidades de intervenção e eventualmente comprometer o efeito do programa.

Como limitação deste estudo que teve um desenho ecológico, destaca-se que as taxas de mortalidade utilizadas no modelo de análise podem ter sido subestimadas em função do registro de óbitos cuja causa foi classificada como mal definida e pela incompletude no preenchimento das declarações de óbito que alimentam o Sistema de Informação sobre Mortalidade (SIM $)^{46}$.

Por outro lado, o presente estudo configurase como inovador, pois utiliza um método amplamente empregado para a avaliação de impacto de políticas sociais, mas que ainda é pouco explorado no campo da saúde. Além disso, as evidências geradas a partir deste estudo podem servir de referência para avaliar o impacto de outros programas de promoção da saúde e da atividade física e subsidiar a tomada de decisão dos gestores públicos no planejamento de estratégias direcionadas à implantação ou expansão do Programa Academia da Saúde em seus municípios. 


\section{Colaboradores}

FRB Guarda contribuiu na concepção e delineamento do estudo, redação do manuscrito, interpretação e análises de dados, até a revisão final do manuscrito. DKS Feitosa participou da redação do manuscrito, obtenção, interpretação e análises de dados, até a revisão final do manuscrito. RN Silva participou da redação, análise crítica e revisão final do manuscrito. BLS Rodrigues participou da redação do manuscrito, obtenção, interpretação e análises de dados, até a revisão final do manuscrito. PBC Silva contribuiu na redação do manuscrito, obtenção das bases de dados, até a revisão final do manuscrito. RG Arruda contribuiu com a interpretação e análise dos dados, e análise crítica do manuscrito.

\section{Referências}

1. Kearney PM, Whelton M, Reynolds K, Muntner P, Whelton PK, He J. Global Burden of Hypertension: Analysis of Worldwide Data. Lancet 2005; 365(9455):217-223.

2. Malta D, Scala LCN, Fuchs S. Conceituação, Epidemiologia e Prevenção Primária. In: Plavnik FL. $7^{a} D i-$ retriz Brasileira de hipertensão arterial. Rio de Janeiro: Sociedade Brasileira de Cardiologia; 2016. p. 1-6.

3. World Health Organization(WHO).Globalstatus report on noncommunicable diseases [Internet]. 2014 [acessado 2020 abr 20]. Disponível em: https://apps.who.int/ iris/bitstream/handle/10665/148114/9789241564854_ eng.pdf? sequence $=1$.

4. Soares GP, Brum JD, Oliveira GMM, Klein CH, Silva NAS. Evolution of socioeconomic indicators and cardiovascular mortality in three Brazilian states. Arq Bras Cardiol 2013; 100(2):147-156.

5. Lotufo PA, Fernandes TG, Bando DH. Alencar AP, Benseñor IM. Income and heart disease mortality trends in Sao Paulo, Brazil, 1996 to 2010. Int J Cardiol 2013; 167(6):2820-2823.

6. Villela PB, Klein CH, Oliveira GMM. Socioeconomic factors and mortality due to cerebrovascular and hypertensive disease in Brazil. Rev Port Cardiol 2019; 38(3):205-212.

7. Guimarães RM, Andrade SS, Machado EL, Bahia CA, Oliveira MM, Jacques FV. Regional differences in cardiovascular mortality transition in Brazil. 1980 to 2012. Rev Panam Salud Publica 2015; 37(2):83-89.

8. Chor D, Ribeiro ALP, Carvalho MS, Duncan BB, Lotufo PA, Nobre AA, Aquino EMLL, Schmidt MI, Griep RH, Molina MDCB, Barreto SM, Passos VMA, Benseñor IJM, Matos SMA, Mill JG. Prevalence. awareness. treatment and influence of socioeconomic variables on control of high blood pressure: results of the ELSA Brasil Study. PLoS One 2015; 10(6):e0127382.

9. Santos MAA, Prado BS, Santos DMS. Análise Espacial e Tendências de Mortalidade Associada a Doenças Hipertensivas nos Estados e Regiões do Brasil entre 2010 e 2014. Int J Cardiovasc Sci 2018; 31(3)250-257.

10. Andrade SSA, Stopa SR, Brito AS, Chueri OS, Szwarcwald CL, Malta DC. Prevalência de hipertensão arterial autorreferida na população brasileira: análise da Pesquisa Nacional de Saúde. 2013. Epidemiol Serv Saude 2015; 24(2):297-304.

11. Lear SA, Hu W, Rangarajan S, Gasevic D, Leong D, Iqbal R, Casanova A, Swaminathan S, Anjana RM, Kumar R, Rosengren A, Wei L, Yang W, Chuangshi W, Huaxing L, Nair S, Diaz R, Swidon H, Gupta R, Mohammadifard N, Jaramillo PL, Oguz A, Zatonska K, Seron P, Avezum A, Poirier P, Teo K, Yusuf S. The effect of physical activity on mortality and cardiovascular disease in 130.000 people from 17 high-income. middle-income. and low-income countries: the PURE study. Lancet 2017; 390(10113):2643-2654.

12. Leitzmann MF, Park Y, Blair A, Ballard-Barbash R, Mouw T, Hollenbeck AR, Schatzkin A. Physical Activity Recommendations and Decreased Risk of Mortality. Arch Intern Med 2007; 167(22):2453-2460.

13. Brown RE, Riddell MC, Macpherson AK, Canning KL, Kuk JL. The Joint Association of Physical Activity. Blood-Pressure Control and Pharmacologic Treatment of Hypertension for All-Cause Mortality Risk. Am J Hypertens 2013; 26(8):1005-1010. 
14. Kahn EB, Ramsey LT, Brownson RC, Heath GH, Howze EH, Powell KE, Stone EJ, Rajab MW, Corso P. The effectiveness of interventions to increase physical activity. A systematic review. Am J Prev Med 2002; 22(Supl. 4):73-107.

15. Malta DC, Silva JB. Policies to promote physical activity in Brazil. Lancet 2012; 380(9838):195-196.

16. Silva RN, Guarda FRB, Hallal PC, Martelli PJL. Avaliabilidade do Programa Academia da Saúde no Município do Recife, Pernambuco, Brasil. Cad Saude Publica 2017; 33(4):e00159415.

17. Brasil. Portaria no 2.681, de 7 de novembro de 2013. Redefine o Programa Academia da Saúde no âmbito do Sistema Único de Saúde (SUS). Diário Oficial da União 2013; 7 nov.

18. Ivo MAS, Malta DC, Freitas MI. Modos de pensar dos profissionais do Programa Academia da Saúde sobre saúde e doença e suas implicações nas ações de promoção de saúde. Physis 2019; 29(1):e290110.

19. Guarda FRB, Silva RN, Araújo Júnior JLAC, Freitas MIF, Santos Neto PM. Intervenção do profissional de educação física: formação. perfil e competências para atuar no Programa Academia da Saúde. Rev Pan -Amaz Saude 2014; 5(4):63-74.

20. Brasil. Ministério da Saúde (MS). Cadastro Nacional de Estabelecimentos de Saúde (CNES). Distribuição dos polos do Programa Academia da Saúde 2017 [Internet]. [acessado 2020 maio 16]. Disponível em: http://tabnet.datasus.gov.br/cgi/tabcgi.exe?cnes/cnv/ estabbr.def.

21. Pernambuco. Secretaria Estadual de Saúde. Gerência de Expansão e Qualificação da Atenção Primária. Relação dos Municípios Contemplados ao Custeio das Ações do Programa Academia da Saúde [Internet]. 2013 [acessado 2020 maio 16]. Disponível em: http:// geqap.blogspot.com.br/2013/10/monitoramento-nacional-do programa.html

22. Sá GBAR, Dornelles GC, Cruz KG, Amorim RCA, Andrade SSCA, Oliveira TP Silva MMA, Malta DC, Souza MFM. O Programa Academia da Saúde como estratégia de promoção da saúde e modos de vida saudáveis: cenário nacional de implementação. Cien Saude Colet 2016; 21(6):1849-1859.

23. Malta DC, Silva JR. O Plano de Ações Estratégicas para o Enfrentamento das Doenças Crônicas Não Transmissíveis no Brasil e a definição das metas globais para o enfrentamento dessas doenças até 2025: uma revisão. Epidemiol Serv Saude 2013; 22(1):151164.

24. Guarda FRB, Silva RN, Feitosa WMN, Santos Neto PM, Araújo Júnior JLAC. Caracterização das equipes do Programa Academia da Saúde e do seu processo de trabalho. Rev Bras Ativ Fis Saude 2015; 20(6):638-640.

25. Wooldridge JM. Introdução à Econometria: uma abordagem moderna. $3^{\mathrm{a}}$ ed. São Paulo: Cengage Learning; 2016.

26. Khandker SR, Koolwal GB, Samad HA. Handbook on Impact Evaluation: Quantitative Methods and Practices [Internet]. The World Bank; 2010 [acessado 2020 maio 16]. Disponível em: https://openknowledge.worldbank. org/bitstream/handle/10986/2693/520990PUB0EPI1101Official0Use0Only1.pdf? sequence $=1$ \&isAllowed $=\mathrm{y}$.
27. Ramos MP, Lermen JI, Busatto L, Matos J. Avaliação de impacto de Políticas Públicas: uma experiência com Projeto Inverno Gaúcho da Secretaria da Saúde do Estado do Rio Grande. Rev Polit Publicas 2010; 14(2):297-306.

28. Fontes LFC, Conceição OC, Saraiva MV. Três anos do programa mais médicos: uma análise econométrica [Internet]. UFSM; 2016 [acessado 2020 abr 20]. Disponível em: http://coral.ufsm.br/seminarioeconomia/ images/anais_2016/TRS-ANOS-DO-PROGRAMA -MAIS-MDICOS-UMA-ANLISE-ECONOMTRICA. pdf.

29. Pino FA. Modelos de Decisão Binários: uma revisão. Rev Econom Agricol 2007; 54(1):43-57.

30. Liao TF. Interpreting probability models: Logit, probit, and other generalized linear models. Models: 101. Thousand Oaks: Sage; 1994.

31. Austin PC. An Introduction to Propensity Score Methods for Reducing the Effects of Confounding in Observational Studies. Pharm Stat 2011; 46(3):399-424.

32. Brasil. Ministério da Saúde (MS). Cadastro Nacional de Estabelecimentos de Saúde (CNES). Departamento de Informática do SUS. Recursos Físicos - Hospitalar - Leitos De Internação - Brasil. Quantidade SUS por ano/mês competência segundo município. Nos anos de 2010 a 2017 [Internet] [acessado 2020 maio 16]. Disponível em: http://tabnet.datasus.gov.br/cgi/tabcgi. exe?cnes/cnv/leiintbr.def.

33. Neves RG, Flores TR, Duro SMS, Nunes BP, Tomasi E. Tendência temporal da cobertura da Estratégia Saúde da Família no Brasil, regiões e unidades da federação. 2006-2016. Epidemio IServ Saude 2018; 27(3):e2017170.

34. Brant LCC, Nascimento BR, Passos VMA, Duncan BB, Bensenõr IJM, Malta DC, Souza MFM, Ishitani LH, França E, Oliveira MS, Mooney M, Naghavi M, Roth G, Ribeiro ALP. Variações e diferenciais da mortalidade por doença cardiovascular no Brasil e em seus estados, em 1990 e 2015: estimativas do Estudo Carga Global de Doença. Rev Bras Epidemiol 2017; 20(Supl. 1):116-128.

35. Lotufo PA. Bensenor IJM. Race and stroke mortality in Brazil. Rev Saude Publica 2013; 47(6):1201-1204.

36. Francisco PMSB, Segri NJ, Borim FSA, Malta DC. Prevalência simultânea de hipertensão e diabetes em idosos brasileiros: desigualdades individuais e contextuais. Cien Saude Colet 2018; 23(11):3829-3840.

37. Becker SO, Ichino A. Estimation of average treatment effects based on propensity scores. Stata J 2002; 2(4):358-377.

38. Caliendo M, Kopeining S. Some practical guidance for the implementation of propensity score matching. $J$ Econom Surv 2008; 22(1):31-72.

39. Rosenbaum P, Rubin D. The Central Role of the Propensity Score in Observational Studies for Causal Effects. Biometrika 1983; 70(1):41-55.

40. Ravaioli PF. O impacto do programa Bolsa Família na posse de bens duráveis [dissertação]. Piracicaba: USP; 2018.

41. Malta DC, Bernal RTI, Andrade SSCA, Silva MMA, Melendez GV. Prevalência e fatores associados com hipertensão arterial autorreferida em adultos brasileiros. Rev Saude Publica 2017; 51(Supl.):11s. 
42. Esperandio EM, Espinosa MM, Martins MAS, Guimarães LV, Lopes MAL, Scala LCN. Prevalência e fatores associados à hipertensão arterial em idosos de municípios da Amazônia Legal. MT. Rev Bras Geriatr Gerontol 2013;16(3):481-493.

43. Heckman J, Ichimura H, Todd P. Matching as an econometric evaluation estimator: evidence from evaluating a job training program. Rev Econom Stud 1997; 64(4):605-654.

44. Simões EJ, Hallal PC, Siqueira FV, Schmaltz C, Menor D, Malta DC, Duarte H, Hino AA, Mielke GI, Pratt M, Reis RS. Effectiveness of a scaled up physical activity intervention in Brazil: A natural experiment. Prev Med 2016; 103S:S66-S72.

45. Fernandes AP, Andrade ACS, Costa DAS, Dias MAS, Malta DC, Caiaffa WTQ. Programa Academias da Saúde e a promoção da atividade física na cidade: a experiência de Belo Horizonte. MG. Brasil. Cien Saude Colet 2017; 22(12):3903-3914.

46. Morais RM, Costa AL. Uma avaliação do Sistema de Informações sobre Mortalidade. Saude Debate 2017; 41(n. esp.):101-117.

Artigo apresentado em 20/07/2020

Aprovado em 19/10/2020

Versão final apresentada em 21/10/2020

Editores-chefes: Romeu Gomes, Antônio Augusto Moura da Silva 